\title{
Distributed Compressed Sensing for the MIMO MAC with Correlated Sources
}

\author{
Steven Corroy and Rudolf Mathar \\ Institute for Theoretical Information Technology \\ RWTH Aachen University \\ Aachen, Germany \\ \{corroy, mathar\}@ti.rwth-aachen.de
}

\begin{abstract}
In this paper we consider the transmission of jointly sparse signals over a MIMO MAC composed of two transmitters and one receiver. Distributed compression is performed at the transmitters using a liner transformation and joint reconstruction at the receiver is enabled using the theory of distributed compressed sensing. The objective is to minimize the sum MSE between the uncompressed signals at the transmitter and the recovered signals at the receiver. We present a new theoretical framework to study this problem and provide an algorithm enabling to transmit correlated signals over a MIMO MAC, which performs provably close to optimal. To validate our approach we analyze the performance of our system for different noise conditions and varying compression factors. The results show that distributed compressed sensing can be performed reliably over a MIMO MAC.
\end{abstract}

\section{INTRODUCTION}

Multiple-input multiple-output (MIMO) communication has been the main driver of the increased data throughput of mobile communication systems in the recent past years. Nevertheless, the increasing demand for mobile traffic requires novel methods to further improve the efficiency of MIMO systems. One very promising approach to meet this challenge is cooperation. In this paper we consider the MIMO multiple access channel (MIMO MAC), which consists in letting several transmitters access concurrently the same resources. This problem has been extensively studied and we recall here some of the main results. In [1], the authors provide an iterative water-filling algorithm, which achieves the capacity of the MIMO MAC with individual power constraints for each transmitter. In [2], the authors extend the approach of [1] to the case of a global power constraint on all transmitters. While the two previous works consider rate maximization, in [3], the authors derive methods to minimize the sum mean-square error (MSE) given individual power constraints.

These works however do not consider correlated sources and the performance improvement that can be achieved by exploiting this correlation. In [4], the authors examine a setup consisting of one MIMO transmitter communicating in a first step with several MIMO base stations. In a second step, the base stations compress the received signals using distributed

This work was partly supported by the Deutsche Forschungsgemeinschaft (DFG) project CoSeCo (grant MA 1184/18-1) and by the UMIC research cluster of the RWTH Aachen University.
Wyner-Ziv coding and forward them onto a lossless backhaul to a central base station. In [5], the authors consider a similar setup, where a transmitter with several antennas transmits a signal to multiple agents with a single antenna, which distributively compress and forward their received signal onto lossless links with finite capacity. As opposed to these approaches, we want to directly transmit distributively compressed signal over a MIMO MAC in a single step.

The main idea of the present work is to use distributed compressed sensing (DCS) in order to separately compress the source signals, transmit them over a MIMO MAC and finally jointly recover these signals while exploiting their correlation.

DCS is an extension of compressed sensing (CS) [6], which considers the compression and recovery of a global signal $\mathbf{z}$ decomposed in subsignals $\mathbf{z}_{1}, \ldots, \mathbf{z}_{n}$, with $\mathbf{z}=\left[\mathbf{z}_{1}^{\mathrm{T}}, \ldots, \mathbf{z}_{n}^{\mathrm{T}}\right]^{\mathrm{T}}$, which are compressed independently. The global signal is recovered in its entirety at one location. DCS is particularly interesting to compress sources that are correlated but cannot be processed together (e.g., sensors at different locations). A simple setup of DCS for wireless sensor networks has been presented in [7]. Each source $j$, with $j=1, \ldots, n$, wants to transmit a scalar $z_{j} \in \mathbb{C}$ to a sink. At time $t$ each source transmits $\phi_{j}(t) z_{j}$ where $\phi_{j}(t)$ is a random value known at the sink. The sink receives $\sum_{j=1}^{n} \phi_{j}(t) z_{j}+n(t)$. After $m$ channel uses, the sink received $\mathbf{y} \in \mathbb{C}^{m}$ with $\mathbf{y}=\boldsymbol{\Phi} \mathbf{z}+\mathbf{n}, \boldsymbol{\Phi} \in \mathbb{C}^{m \times n}, \boldsymbol{\Phi}_{i j}=\phi_{j}(i), \mathbf{z}=\left[\begin{array}{ll}z_{1} & z_{2} \ldots z_{n}\end{array}\right]^{\mathrm{T}}$ and $\mathbf{n}=[n(1) n(2) \ldots n(m)]^{\mathrm{T}}$. If $\mathbf{z}$ is sparse then it can be reconstructed with $m<n$ using CS theory.

In [7] the main signal is assumed to be compressible, i.e., sparse in some domain. In [8], the authors extend the scope of DCS and define the concept of joint sparsity, which represents the fact that several subsignals have a joint structure. The authors present several joint sparsity models (JSM) which are interesting for different practical scenarios. For example the JSM-1 model, describes subsignals sharing a common sparse component and having an individual sparse innovation component which is, e.g., a model for distributed sensor measurements. The JSMs account both for intra-signal correlation (inside the subsignals) and inter-signal correlation (among subsignals). For each JSM, the authors provide reconstruction algorithms and error bounds. In [9], distributed compressed sensing has been applied to amplified and forward relay 
networks to perform distributed compression of jointly sparse signals. However, in contrast to the present work, correlated signals are not considered (i.e., the common sparse component of the JSM-1 model is set to zero), and the optimization of the transmit strategy ignores the noise in the system.

The contribution of the present paper is manifolds. First we introduce a new theoretical framework to analyze the performance of DCS over a MIMO MAC. Second we provide a lower bound on the achievable sum MSE for the transmission of correlated signals over a MIMO MAC and most importantly we derive a heuristic, which achieves a sum MSE matching, in practice, this lower bound. Finally we verify our methodology by proceeding to numerical analysis and discuss the performance achieved by such a system, as well as the influence of the compression rate on the reconstruction error.

The rest of the present paper is organized as follow. In Section II, we describe the problem statement and our theoretical framework. In Section III we derive a lower bound on the achievable error and present a heuristic to minimize the sum MSE with individual transmit power constraints. In Section IV we show numerical results in order to validate our approach. Finally, section V concludes this work.

\section{System Model and Optimization Problem}

We consider a MIMO MAC with two transmitters and one receiver. Each transmitter $i$ has a source signal $\mathbf{z}_{i} \in \mathbb{C}^{n}$. These source signals are correlated and obey the JSM-1 [8], i.e.,

$$
\mathbf{z}_{i}=\hat{\mathbf{z}}_{i}+\mathbf{z}_{c}, \quad i \in\{1,2\},
$$

where $\hat{\mathbf{z}}_{i}$ is a sparse innovation vector and $\mathbf{z}_{c}$ is a sparse vector common to both sources. A vector is said to be $k$-sparse if it has no more than $k$ nonzero components. In order to analyze our system we need to be able to generate signals $\mathbf{z}_{i}$ obeying the JSM-1. In this work we adopt the following methodology. The entries of the vectors $\hat{\mathbf{z}}_{1}, \hat{\mathbf{z}}_{2}$ and $\hat{\mathbf{z}}_{c}$ are independently and identically-distributed as follows

$$
\begin{array}{ll}
\hat{z}_{i, j} \sim \mathcal{N}_{\mathbb{C}}(0,1), & \text { with probability } p_{i}, \\
\hat{z}_{i, j}=0, & \text { with probability } 1-p_{i}
\end{array}
$$

and

$$
\begin{array}{ll}
z_{c, j} \sim \mathcal{N}_{\mathbb{C}}(0,1), & \text { with probability } p_{c}, \\
z_{c, j}=0, & \text { with probability } 1-p_{c},
\end{array}
$$

where $\hat{z}_{i, j}$ and $z_{c, j}$ are the $j$-th component of $\hat{\mathbf{z}}_{i}$ and $\mathbf{z}_{c}$ respectively. Note that $\hat{z}_{i, j}$ and $z_{c, j}$ are $p_{i} n$-sparse and $p_{c} n$ sparse respectively in average, i.e., of the $n$ entries, no more than $p_{i} n$ and $p_{c} n$ are nonzero in average respectively.

Each transmitter $i$ has $m$ antennas. It compresses its source signal using a Gaussian matrix $\boldsymbol{\Phi}_{i} \in \mathbb{R}^{m \times n}$ with $m<n$ and all entries of $\boldsymbol{\Phi}_{i}$ are independently and identically-distributed as $\left[\boldsymbol{\Phi}_{i}\right]_{j k} \sim \mathcal{N}_{\mathbb{C}}(0,1 / m)$. Note that the matrices $\boldsymbol{\Phi}_{i}$ are generated once and are fixed and known to the transmitter and the receiver in the following. The signals $\mathbf{x}_{i} \in \mathbb{R}^{m}$ resulting from this compression are given by

$$
\mathbf{x}_{i}=\boldsymbol{\Phi}_{i} \mathbf{z}_{i}, \quad i \in\{1,2\} .
$$

The entries of $\mathbf{x}_{i}$ have zero mean and the covariance matrix $\mathbf{C}_{i}$ of $\mathbf{x}_{i}$ is given by

$$
\mathbf{C}_{i}=\left(p_{i}+p_{c}\right) \boldsymbol{\Phi}_{i} \boldsymbol{\Phi}_{i}^{\mathrm{H}}, \quad i \in\{1,2\} .
$$

Similarly the cross-correlation matrix $\mathbf{C}_{12}$ between $\mathbf{x}_{1}$ and $\mathbf{x}_{2}$ is given by

$$
\mathbf{C}_{12}=p_{c} \boldsymbol{\Phi}_{1} \boldsymbol{\Phi}_{2}^{\mathrm{H}}, \quad i \in\{1,2\} .
$$

The signals $\mathbf{x}_{i}$ are precoded with matrices $\mathbf{P}_{i} \in \mathbb{C}^{m \times m}$ and transmitted over the wireless channel. The received signal $\mathbf{y} \in \mathbf{C}^{m}$ is given by

$$
\mathbf{y}=\mathbf{H}_{1} \mathbf{P}_{1} \mathbf{x}_{1}+\mathbf{H}_{2} \mathbf{P}_{2} \mathbf{x}_{2}+\mathbf{n},
$$

where $\mathbf{H}_{i} \in \mathbf{C}^{m \times m}$ is a channel gain matrix and $\mathbf{n} \in \mathbb{C}^{m}$ is a noise vector with entries taken from the Gaussian distribution as $\mathbf{n} \sim \mathcal{N}_{\mathbb{C}}\left(0, \mathbf{C}_{\mathbf{n}}\right)$. In the present work, we consider a global power constraint on the two transmitters, i.e., $\operatorname{tr}\left(\mathbf{P}_{1} \mathbf{C}_{1} \mathbf{P}_{1}^{\mathrm{H}}+\right.$ $\left.\mathbf{P}_{2} \mathbf{C}_{2} \mathbf{P}_{2}^{\mathrm{H}}\right) \leq P_{T}$.

By defining $\mathbf{x} \triangleq\left[\left(\mathbf{P}_{1} \mathbf{x}_{1}\right)^{\mathrm{T}}\left(\mathbf{P}_{2} \mathbf{x}_{2}\right)^{\mathrm{T}}\right]^{\mathrm{T}}, \mathbf{z} \triangleq\left[\mathbf{z}_{1}^{\mathrm{T}} \mathbf{z}_{2}^{\mathrm{T}}\right]^{\mathrm{T}}$,

$$
\mathbf{H} \triangleq\left[\begin{array}{ll}
\mathbf{H}_{1} & \mathbf{H}_{2}
\end{array}\right], \mathbf{P} \triangleq\left[\begin{array}{cc}
\mathbf{P}_{1} & \mathbf{0} \\
\mathbf{0} & \mathbf{P}_{2}
\end{array}\right] \text { and } \mathbf{\Phi} \triangleq\left[\begin{array}{cc}
\boldsymbol{\Phi}_{1} & \mathbf{0} \\
\mathbf{0} & \mathbf{\Phi}_{2}
\end{array}\right] \text {, }
$$

we can rewrite (7) as follows

$$
\mathbf{y}=\mathbf{H x}+\mathbf{n}=\mathbf{H P \Phi z}+\mathbf{n} .
$$

The signal $\mathbf{y}$ is linearly filtered by a filter matrix $\mathbf{W} \in \mathbb{C}^{2 m \times m}$. The final filtered signal $\tilde{\mathbf{x}} \in \mathbb{C}^{2 m}$ is given by

$$
\tilde{\mathbf{x}}=\mathbf{W}^{\mathrm{H}} \mathbf{y} .
$$

Finally the receiver tries to recover $\mathbf{z}_{1}$ and $\mathbf{z}_{2}$ using DCS by solving the following convex program

$$
\begin{aligned}
& \underset{\hat{\mathbf{z}}_{1}, \hat{\mathbf{z}}_{2}, \mathbf{z}_{c}}{\operatorname{minimize}}\left\|\hat{\mathbf{z}}_{2}\right\|_{l_{1}}+\left\|\hat{\mathbf{z}}_{1}\right\|_{l_{1}}+\left\|\mathbf{z}_{c}\right\|_{l_{1}} \\
& \text { subject to }\left\|\mathbf{W}^{\mathrm{H}} \mathbf{H P \Phi}\left[\begin{array}{l}
\hat{\mathbf{z}}_{1}+\mathbf{z}_{c} \\
\hat{\mathbf{z}}_{2}+\mathbf{z}_{c}
\end{array}\right]-\tilde{\mathbf{x}}\right\|_{l_{2}} \leq \sqrt{\operatorname{tr}\left(\mathbf{W}^{\mathrm{H}} \mathbf{C}_{\mathbf{n}} \mathbf{W}\right)},
\end{aligned}
$$

where $\sqrt{\operatorname{tr}\left(\mathbf{W}^{\mathrm{H}} \mathbf{C}_{\mathbf{n}} \mathbf{W}\right)}$ is the average norm of the received noise, $\|\cdot\|_{l_{1}}$ denotes the $l_{1}$-norm, i.e., the sum of the absolute value of the entries of the vector and $\|\cdot\|_{l_{2}}$ denotes the Euclidean norm. The constraint of the problem amounts to only taking into account signals $\mathbf{z}_{1}$ and $\mathbf{z}_{2}$, which are consistent with the received vector $\tilde{\mathbf{x}}$. The objective then chooses among all possible consistent signals, the ones, which are the sparsest. Indeed, the $l_{1}$-norm is the tightest convex approximation of the cardinality function.

The fundamental questions that are of interest in the present work, are 1) how to design the matrices $\mathbf{P}_{1}, \mathbf{P}_{2}$ and $\mathbf{W}$ to minimize the MSE between $\mathbf{x}$ and $\tilde{\mathbf{x}}$ by choosing $\mathbf{P}_{1}, \mathbf{P}_{2}$ and $\mathbf{W}$, while fulfilling the power constraint

$$
\operatorname{tr}\left(\mathbf{P}_{1} \mathbf{C}_{1} \mathbf{P}_{1}^{\mathrm{H}}+\mathbf{P}_{2} \mathbf{C}_{2} \mathbf{P}_{2}^{\mathrm{H}}\right) \leq P_{T},
$$

2) how small can $m$ be chosen, in other words how much antenna should we have for a given $n$ and 3) what performance can be achieved. 


\section{Filter AND PRecoder Design}

The contribution of this section is to extend the approach of [3] to colored noise and, most important, to correlated sources. The MSE matrix, denoted by $\mathbf{E} \in \mathbb{R}^{2 n \times 2 n}$, is given by $\mathbf{E}=\mathbb{E}\left[(\tilde{\mathbf{x}}-\mathbf{x})(\tilde{\mathbf{x}}-\mathbf{x})^{\mathrm{H}}\right]$. We define the matrix $\mathbf{Q}$ as the covariance matrix of $\mathbf{x}$, i.e.,

$$
\mathbf{Q} \triangleq\left[\begin{array}{cc}
\mathbf{P}_{1} \mathbf{C}_{1} \mathbf{P}_{1}^{\mathrm{H}} & \mathbf{P}_{1} \mathbf{C}_{12} \mathbf{P}_{2}^{\mathrm{H}} \\
\mathbf{P}_{2} \mathbf{C}_{12}^{\mathrm{H}} \mathbf{P}_{1}^{\mathrm{H}} & \mathbf{P}_{2} \mathbf{C}_{2} \mathbf{P}_{2}^{\mathrm{H}}
\end{array}\right] .
$$

We start with $\mathbf{Q}$ as our optimization variable without considering its structure given in (12) and come back to this point later in our derivations. We want to solve the following optimization problem

$$
\begin{array}{ll}
\underset{\mathbf{Q}, \mathbf{W}}{\operatorname{minimize}} & \operatorname{tr}\left(\mathbf{Q}^{-\frac{1}{2}} \mathbf{E} \mathbf{Q}^{-\frac{1}{2}}\right) \\
\text { subject to } & \operatorname{tr}(\mathbf{Q}) \leq P_{T} \\
& \mathbf{Q} \succcurlyeq \mathbf{0},
\end{array}
$$

where $\mathbf{E}$ is given by

$$
\begin{aligned}
& \mathbf{E}=\mathbf{W}^{\mathrm{H}}\left(\mathbf{H} \mathbf{Q} \mathbf{H}^{\mathrm{H}}+\mathbf{C}_{\mathbf{n}}\right) \mathbf{W}-\mathbf{W}^{\mathrm{H}} \mathbf{H Q}-\mathbf{Q} \mathbf{H}^{\mathrm{H}} \mathbf{W}+\mathbf{Q} \text {. } \\
& \text { and } \operatorname{tr}\left(\mathbf{Q}^{-\frac{1}{2}} \mathbf{E} \mathbf{Q}^{-\frac{1}{2}}\right) \text { is the normalized MSE by } \mathbf{Q} \text {. }
\end{aligned}
$$

\section{A. Optimal Filter Design}

It can be easily shown that the minimum MSE filter matrix $\mathbf{W}^{\star}$ is given by

$$
\mathbf{W}^{\star}=\left(\mathbf{H Q H} \mathbf{H}^{\mathrm{H}}+\mathbf{C}_{\mathbf{n}}\right)^{-1} \mathbf{H Q} .
$$

By plugging (15) into (14), we get the MSE matrix with optimal filtering given by

$$
\mathbf{E}=\mathbf{Q}-\mathbf{Q H}^{\mathrm{H}}\left(\mathbf{H Q} \mathbf{H}^{\mathrm{H}}+\mathbf{C}_{\mathbf{n}}\right)^{-1} \mathbf{H} \mathbf{Q} .
$$

\section{B. Optimal Precoder Design}

We can rewrite the objective function of the problem (13) as follows

$$
\begin{aligned}
\operatorname{tr}\left(\mathbf{Q}^{-\frac{1}{2}} \mathbf{E} \mathbf{Q}^{-\frac{1}{2}}\right) & =\operatorname{tr}\left(\mathbf{I}-\mathbf{Q}^{\frac{1}{2}} \mathbf{H}^{\mathrm{H}}\left(\mathbf{H} \mathbf{H} \mathbf{H}^{\mathrm{H}}+\mathbf{C}_{\mathbf{n}}\right)^{-1} \mathbf{H} \mathbf{Q}^{\frac{1}{2}}\right) \\
& =m+\operatorname{tr}\left(\left(\mathbf{H} \mathbf{Q} \mathbf{H}^{\mathrm{H}}+\mathbf{C}_{\mathbf{n}}\right)^{-1} \mathbf{C}_{\mathbf{n}}\right) \\
& =m+\operatorname{tr}\left(\left(\mathbf{C}_{\mathbf{n}}{ }^{-\frac{1}{2}} \mathbf{H} \mathbf{Q} \mathbf{H}^{\mathrm{H}} \mathbf{C}_{\mathbf{n}}{ }^{-\frac{1}{2}}+\mathbf{I}\right)^{-1}\right)
\end{aligned}
$$

We can now find the optimal value for $\mathbf{Q}$, denoted $\mathbf{Q}^{\star}$ by solving the following convex optimization problem

$$
\begin{array}{ll}
\underset{\mathbf{Q}}{\operatorname{minimize}} & \operatorname{tr}\left(\left(\mathbf{C}_{\mathbf{n}}{ }^{-\frac{1}{2}} \mathbf{H} \mathbf{Q} \mathbf{H}^{\mathrm{H}} \mathbf{C}_{\mathbf{n}}{ }^{-\frac{1}{2}}+\mathbf{I}\right)^{-1}\right) \\
\text { subject to } & \operatorname{tr}(\mathbf{Q}) \leq P_{T} \\
& \mathbf{Q} \succcurlyeq \mathbf{0} .
\end{array}
$$

Now, the problem is, as mentioned earlier, that we do not have the complete freedom for choosing $\mathbf{Q}$ since the two transmitters precode independently their own signal. We make explicit the block structure of $\mathbf{Q}$ as follows

$$
\mathbf{Q}=\left[\begin{array}{cc}
\mathbf{Q}_{1} & \mathbf{Q}_{12} \\
\mathbf{Q}_{12}^{\mathrm{H}} & \mathbf{Q}_{2}
\end{array}\right]
$$

By comparing this expression for $\mathbf{Q}$ to the one in (12), we can see that it is only possible to generate a matrix $\mathbf{Q}$ with the precoders $\mathbf{P}_{1}$ and $\mathbf{P}_{2}$ if the following system has a solution

$$
\left\{\begin{array}{l}
\mathbf{P}_{1} \mathbf{C}_{1} \mathbf{P}_{1}^{\mathrm{H}}=\mathbf{Q}_{1} \\
\mathbf{P}_{2} \mathbf{C}_{2} \mathbf{P}_{2}^{\mathrm{H}}=\mathbf{Q}_{2} \\
\mathbf{P}_{1} \mathbf{C}_{12} \mathbf{P}_{2}^{\mathrm{H}}=\mathbf{Q}_{12} .
\end{array}\right.
$$

Definition 1. A matrix $\mathbf{Q}$ is said to be generable if and only if it there exist precoder matrices $\mathbf{P}_{1}$ and $\mathbf{P}_{2}$ solutions to the system (20).

If we could calculate $\mathbf{Q}^{\star}$ using problem (18) and if there would exist matrices $\mathbf{P}_{1}$ and $\mathbf{P}_{2}$ solving the system (20), then we would have our optimal $\mathbf{P}_{1}$ and $\mathbf{P}_{2}$. The problem is that $\mathbf{Q}^{\star}$ will most often not be generable using $\mathbf{P}_{1}$ and $\mathbf{P}_{2}$. So instead of computing $\mathbf{Q}^{\star}$ and hoping it is generable, we would like to find the best $\mathbf{Q}$ for problem (18) with the constraint that $\mathbf{Q}$ is generable, i.e., find the best signal covariance matrix among those we can actually achieve.

Proposition 1. Define $\mathbf{R}_{\mathbf{c}} \triangleq \mathbf{C}_{1}^{-\frac{1}{2}} \mathbf{C}_{12} \mathbf{C}_{2}^{-\frac{1}{2}}$. A matrix $\mathbf{Q}$ is generable if and only if there exists two orthogonal matrices $\mathbf{U}_{1}$ and $\mathbf{U}_{2}$ such that

$$
\mathbf{Q}_{1}^{\frac{1}{2}} \mathbf{U}_{1}^{\mathrm{H}} \mathbf{R}_{\mathbf{c}} \mathbf{U}_{2} \mathbf{Q}_{2}^{\frac{1}{2}}=\mathbf{Q}_{12}
$$

holds.

Proof: First we show that if $\mathbf{Q}$ is generable then (21) holds. Since $\mathbf{Q}_{1}$ and $\mathbf{C}_{1}$ are symmetric positive semidefinite matrices, all the solutions to $\mathbf{P}_{1} \mathbf{C}_{1} \mathbf{P}_{1}^{\mathrm{H}}=\mathbf{Q}_{1}$ are of the form $\mathbf{P}_{1} \mathbf{C}_{1}^{\frac{1}{2}} \mathbf{U}_{1}=\mathbf{Q}_{1}^{\frac{1}{2}}$ where $\mathbf{U}_{1}$ can be any orthogonal matrix. Similarly all the solutions to $\mathbf{P}_{2} \mathbf{C}_{2} \mathbf{P}_{2}^{\mathrm{H}}=\mathbf{Q}_{2}$ are of the form $\mathbf{P}_{2} \mathbf{C}_{2}^{\frac{1}{2}} \mathbf{U}_{2}=\mathbf{Q}_{2}^{\frac{1}{2}}$. Plugging those two results into the expression $\mathbf{P}_{1} \mathbf{C}_{12} \mathbf{P}_{2}^{\mathrm{H}}=\mathbf{Q}_{12}$, we find that $\mathbf{Q}_{1}^{\frac{1}{2}} \mathbf{U}_{1}^{\mathrm{H}} \mathbf{C}_{1}^{-\frac{1}{2}} \mathbf{C}_{12} \mathbf{C}_{2}^{-\frac{1}{2}} \mathbf{U}_{2} \mathbf{Q}_{2}^{\frac{1}{2}}=\mathbf{Q}_{12}$ must hold.

Second we show that if (21) holds for some matrices $\mathbf{U}_{1}$ and $\mathbf{U}_{2}$, then $\mathbf{Q}$ must be generable, i.e., we can find a solution to the system (20). Simply take the matrices $\mathbf{P}_{1}=\mathbf{Q}_{1}^{\frac{1}{2}} \mathbf{U}_{1}^{\mathrm{H}} \mathbf{C}_{1}^{-\frac{1}{2}}$ and $\mathbf{P}_{2}=\mathbf{Q}_{2}^{\frac{1}{2}} \mathbf{U}_{2}^{\mathrm{H}} \mathbf{C}_{2}^{-\frac{1}{2}}$. We have directly that the first two conditions of (20) are fulfilled. Furthermore since $\mathbf{Q}_{1}^{\frac{1}{2}} \mathbf{U}_{1}^{\mathrm{H}} \mathbf{R}_{\mathbf{c}} \mathbf{U}_{2} \mathbf{Q}_{2}^{\frac{1}{2}}=\mathbf{Q}_{12}$ holds, we can calculate that $\mathbf{Q}_{12}$ equals $\mathbf{P}_{1} \mathbf{C}_{12} \mathbf{P}_{2}^{\mathrm{H}}$, which is exactly the last condition of (20).

The problem to minimize the MSE over all generable covariance matrices can therefore be formulated as follows

$$
\begin{array}{cl}
\underset{\mathbf{Q}, \mathbf{U}_{1}, \mathbf{U}_{2}}{\operatorname{minimize}} & \operatorname{tr}\left(\left(\mathbf{C}_{\mathbf{n}}{ }^{-\frac{1}{2}} \mathbf{H} \mathbf{Q} \mathbf{H}^{\mathrm{H}} \mathbf{C}_{\mathbf{n}}{ }^{-\frac{1}{2}}+\mathbf{I}\right)^{-1}\right) \\
\text { subject to } & \operatorname{tr}(\mathbf{Q}) \leq P_{T} \\
& \mathbf{Q} \succcurlyeq \mathbf{0} \\
& \mathbf{Q}_{1}^{\frac{1}{2}} \mathbf{U}_{1}^{\mathrm{H}} \mathbf{R}_{\mathbf{c}} \mathbf{U}_{2} \mathbf{Q}_{2}^{\frac{1}{2}}=\mathbf{Q}_{12} \\
& \mathbf{U}_{1} \mathbf{U}_{1}^{\mathrm{H}}=\mathbf{I}, \mathbf{U}_{2} \mathbf{U}_{2}^{\mathrm{H}}=\mathbf{I} .
\end{array}
$$

This problem is however nonconvex and hard to solve. We propose next a suboptimal heuristic to solve this problem, which works close to optimal in practice. 


\section{Heuristic for Precoder Design}

In this section we describe an iterative algorithm to solve problem (22).

1) Initialization: The variable $\mathbf{Q}$ is determined by $\mathbf{Q}_{\mathbf{1}}$, $\mathbf{Q}_{2}$ and $\mathbf{Q}_{12}$ as described in (19). We initialize the matrix $\mathbf{Q}_{12}=\mathbf{Q}_{12}^{\star}$, where $\mathbf{Q}_{12}^{\star}$ is the corresponding submatrix of the solution of problem (18).

2) Step 1, find $\mathbf{Q}_{\mathbf{1}}$ and $\mathbf{Q}_{\mathbf{2}}$ : Solve the following convex problem, for which $\mathbf{Q}_{12}$ is fixed,

$$
\begin{array}{ll}
\underset{\mathbf{Q}_{\mathbf{1}}, \mathbf{Q}_{\mathbf{2}}}{\operatorname{minimize}} & \operatorname{tr}\left(\left(\mathbf{C}_{\mathbf{n}}{ }^{-\frac{1}{2}} \mathbf{H} \mathbf{Q} \mathbf{H}^{\mathrm{H}} \mathbf{C}_{\mathbf{n}}{ }^{-\frac{1}{2}}+\mathbf{I}\right)^{-1}\right) \\
\text { subject to } & \operatorname{tr}(\mathbf{Q}) \leq P_{T} \\
& \mathbf{Q} \succcurlyeq \mathbf{0} .
\end{array}
$$

Note that for the first iteration the solution of this problem is the same as the one of problem (18). At this stage $\mathbf{Q}$ does not fulfill the condition (21).

3) Step 2, find $\mathbf{Q}_{12}, \mathbf{U}_{1}$ and $\mathbf{U}_{2}$ : For the first iteration we fix $\mathbf{U}_{2}=\mathbf{I}$ and then solve the following convex problem where $\mathbf{Q}_{\mathbf{1}}$ and $\mathbf{Q}_{\mathbf{2}}$ are fix.

$$
\begin{array}{ll}
\underset{\mathbf{Q}_{12}, \mathbf{U}_{1}}{\operatorname{minimize}} & \operatorname{tr}\left(\left(\mathbf{C}_{\mathbf{n}}{ }^{-\frac{1}{2}} \mathbf{H} \mathbf{Q} \mathbf{H}^{\mathrm{H}} \mathbf{C}_{\mathbf{n}}{ }^{-\frac{1}{2}}+\mathbf{I}\right)^{-1}\right) \\
\text { subject to } & \mathbf{Q} \succcurlyeq \mathbf{0} \\
& \mathbf{Q}_{1}^{\frac{1}{2}} \mathbf{U}_{1}^{\mathrm{H}} \mathbf{R}_{\mathbf{c}} \mathbf{U}_{2} \mathbf{Q}_{2}^{\frac{1}{2}}=\mathbf{Q}_{12} .
\end{array}
$$

In this problem, the optimal value $\mathbf{U}_{1}^{\star}$ is not orthogonal since we dropped the constraint $\mathbf{U}_{1} \mathbf{U}_{1}^{\mathrm{H}}=\mathbf{I}$. To make $\mathbf{U}_{1}$ feasible for problem (22), we take the singular value decomposition (SVD) of $\mathbf{U}_{1}^{\star}$ as $\mathbf{U}_{1}^{\star}=\mathbf{X}_{1} \boldsymbol{\Sigma}_{1} \mathbf{Y}_{1}^{\mathrm{H}}$ and set $\mathbf{U}_{1}$ to the closest orthogonal matrix to $\mathbf{U}_{1}^{\star}$, i.e., $\mathbf{U}_{1}=\mathbf{X}_{1} \mathbf{Y}_{1}^{\mathrm{H}}$.

Second, to find $\mathbf{U}_{2}$ and $\mathbf{Q}_{12}$, we solve the following convex problem

$$
\begin{array}{ll}
\underset{\mathbf{Q}_{12}, \mathbf{U}_{2}}{\operatorname{minimize}} & \operatorname{tr}\left(\left(\mathbf{C}_{\mathbf{n}}{ }^{-\frac{1}{2}} \mathbf{H} \mathbf{Q} \mathbf{H}^{\mathrm{H}} \mathbf{C}_{\mathbf{n}}{ }^{-\frac{1}{2}}+\mathbf{I}\right)^{-1}\right) \\
\text { subject to } & \mathbf{Q} \succcurlyeq \mathbf{0} \\
& \mathbf{Q}_{1}^{\frac{1}{2}} \mathbf{U}_{1}^{\mathrm{H}} \mathbf{R}_{\mathbf{c}} \mathbf{U}_{2} \mathbf{Q}_{2}^{\frac{1}{2}}=\mathbf{Q}_{12} .
\end{array}
$$

Here again $\mathbf{U}_{2}^{\star}$ is not orthogonal. As for $\mathbf{U}_{1}$, we set $\mathbf{U}_{2}=\mathbf{X}_{2} \mathbf{Y}_{2}^{\mathrm{H}}$. Finally it remains to make $\mathbf{Q}_{12}$ feasible by setting it to $\mathbf{Q}_{12}=\mathbf{Q}_{1}^{\frac{1}{2}} \mathbf{U}_{1}^{\mathrm{H}} \mathbf{R}_{\mathbf{c}} \mathbf{U}_{2} \mathbf{Q}_{2}^{\frac{1}{2}}$. The iteration is finished and we go back to Step 1 until the algorithm converges. In practice the convergence happens after very few iterations, typically two or three.

\section{Numerical Evaluation}

We simulate a system with the parameters described in Table I to evaluate the performance of the filter and precoders design presented in Section III. The channel matrix $\mathbf{H}$ is taken

TABLE I

SYSTEM PARAMETERS FOR EVALUATING SUM MSE VS. SNR

\begin{tabular}{lcc}
\hline Parameter & Symbol & Value \\
\hline Size of uncompressed signal $\mathbf{z}_{i}$ & $n$ & 16 \\
Size of compressed signal $\mathbf{x}_{i}$ & $m$ & 4 to 12 \\
Probabilities of nonzero entries in $\mathbf{z}_{i}$ & $p_{1}, p_{2}, p_{c}$ & 0.1 \\
Noise covariance matrix & $\mathbf{C}_{n}$ & $\mathbf{I}$ \\
SNR & $P_{T} / \operatorname{tr}\left(\mathbf{C}_{n}\right)$ & $-20 \mathrm{~dB}$ to $40 \mathrm{~dB}$ \\
\hline
\end{tabular}

at random from the Gaussian distribution.

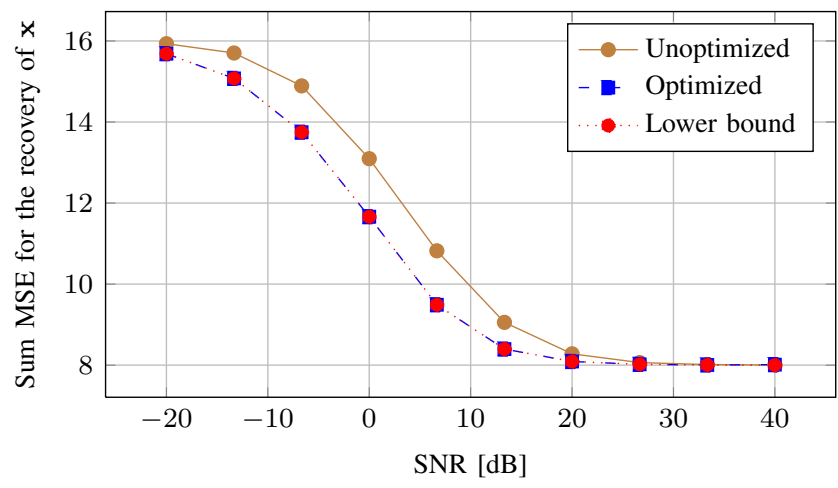

Fig. 1. Theoretical average Sum MSE of compressed vectors with respect to SNR for $m=8$.

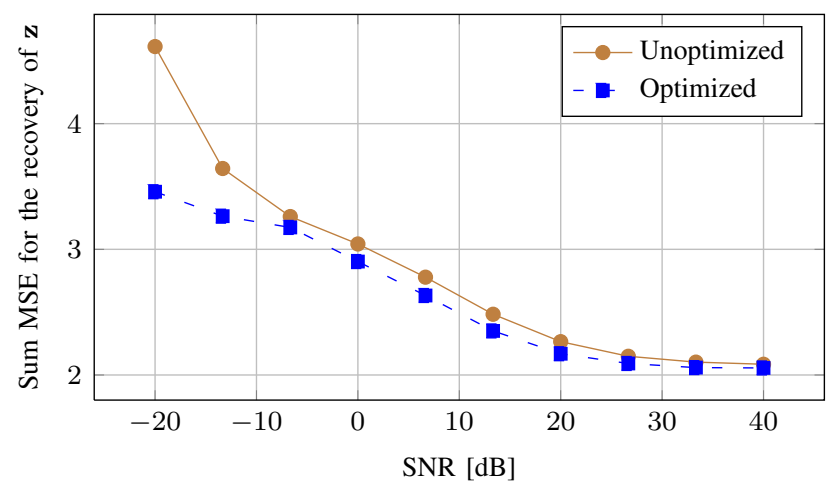

Fig. 2. Experimental average Sum MSE of decompressed vectors with respect to SNR for $m=8$.

\section{A. Sum MSE with respect to SNR}

In this section we want to evaluate the performance of the system under different SNR conditions. In Fig. 1, we plot the theoretical average normalized MSE, between the compressed vectors $\tilde{\mathbf{x}}$ and $\mathbf{x}$, given by formula (17). In dotted red, we present a lower bound on the achievable sum MSE, obtained by solving problem (18), in dashed blue is the sum MSE we achieve using our optimization method described in Section III-C and in solid brown is the sum MSE attained by simply taking $\mathbf{P}_{1}=\mathbf{I}$ and $\mathbf{P}_{2}=\mathbf{I}$, i.e., the communication is not optimized. This figure solely describes the performance of the MIMO MAC. First we can see that our optimization method always achieves performance close to the optimal sum MSE and second we can observe that the gain of optimizing is rather small in very low and high SNR regime but can be as high as $15 \%$ for moderate SNR.

In Fig. 2 and 3, we plot the experimental average MSE obtained by generating several vectors $\mathbf{z}$ as described in (2) and (3), and decompressing using algorithm (10). In solid brown and dashed blue, we can see the performance for an unoptimized and optimized system, respectively. In Fig. 2, we took $m=8$, i.e., a strong compression factor of $1 / 2$ and in Fig. 3, we took $m=12$, i.e., a moderate compression factor of 3/4. Observe the difference between the unoptimized and optimized case. For high compression rate (Fig. 2), we can see a large gain for very low SNR (close to $30 \%$ ) and a small 


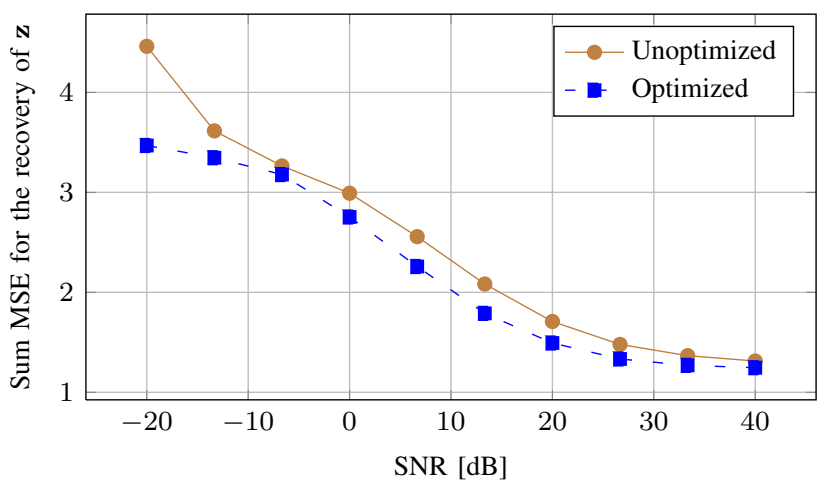

Fig. 3. Experimental average Sum MSE of decompressed vectors with respect to SNR for $m=12$.

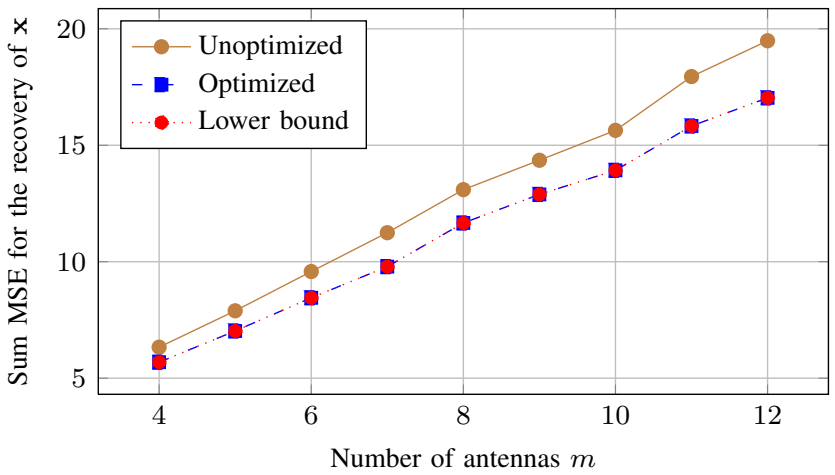

Fig. 4. Theoretical average Sum MSE of compressed vectors with respect to $m$ for $\mathrm{SNR}=0 \mathrm{~dB}$.

gain at moderate SNR. The two curves converge at high SNR. Interestingly, for moderate compression rate (Fig. 3), the gain at high SNR is smaller but the gain at moderate SNR increases significantly (up to $15 \%$ ).

Most importantly these figures show that it is possible to perform distributed compressed sensing reliably over a MIMO MAC. Note that $\hat{\mathbf{z}}_{1}, \hat{\mathbf{z}}_{2}$ and $\mathbf{z}_{c}$, are in average 1.6sparse. In the moderate to high SNR regime (from 20 to $40 \mathrm{~dB}$ ), for a moderate compression rate, we achieve an error, which is smaller than 1.5. It turns out that we reconstruct the common sparse component $\mathbf{z}_{c}$ and one of the sparse innovation component $\hat{\mathbf{z}}_{i}$ perfectly. As the SNR grows, we reconstruct the last innovation component with less and less error.

\section{B. Influence of the Number of Antennas}

In this section we want to transmit a vector $\mathbf{z}$ of fixed size $(2 n)$ and evaluate the performance of the system for different number of antennas $(m)$. In Fig. (4), we plot the theoretical average normalized MSE, between the compressed vectors $\tilde{\mathbf{x}}$ and $\mathbf{x}$ with respect to $m$. In this case again, our optimization algorithm attains the lower bound. For high compression rate, the gain of optimizing is rather small but becomes always larger when the compression factor becomes more moderate. It is important to understand that the error between $\tilde{\mathbf{x}}$ and $\mathbf{x}$ increases with $m$ since the vectors have an increasing number of entries. This does no go against the intuition that compressing $\mathbf{z}$ less, i.e., taking $m$ large, should provide less

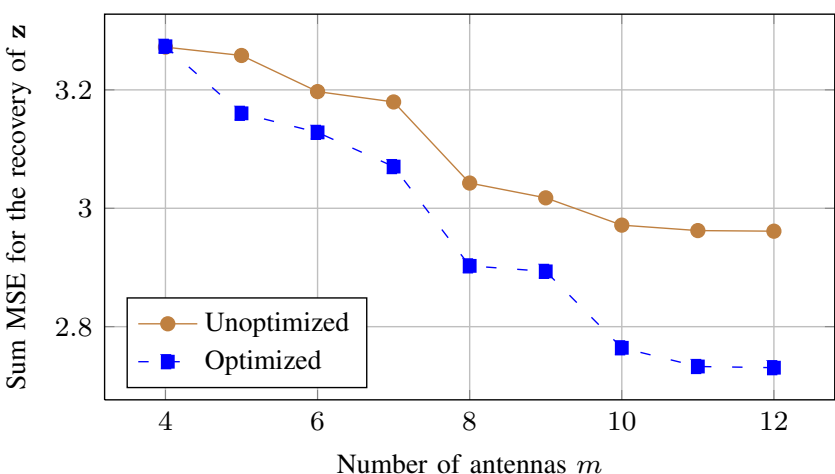

Fig. 5. Experimental average Sum MSE of decompressed vectors with respect to $m$ for $\mathrm{SNR}=0 \mathrm{~dB}$.

error on the reconstruction of $\mathbf{z}$. Indeed in Fig. (5), we plot the reconstruction error of $\mathbf{z}$ with respect to $m$. We clearly see that as $m$ increases, the reconstruction error decreases. Another important point is that as $m$ becomes large, the difference between the unoptimized and optimized case becomes always larger.

\section{CONCLUSION}

We have presented a new theoretical framework enabling to analyze the performance of distributed compressed sensing over the MIMO MAC. In our approach, we first compress separately the correlated signals and then transmit them close to optimal over the MIMO MAC, using a new optimization algorithm. We have shown that our method gives significant improvement over a simple communication strategy and furthermore that distributed compressed sensing can be performed reliably over the MIMO MAC. Future works consist in considering different JSMs and implementing our concept on a cognitive radio platform to verify experimentally our results.

\section{REFERENCES}

[1] W. Yu, W. Rhee, S. Boyd, and J. Cioffi, "Iterative water-filling for Gaussian vector multiple-access channels," IEEE Transactions on Information Theory, vol. 50, no. 1, pp. 145-152, 2004.

[2] H. Boche and E. Jorswieck, "Sum capacity optimization of the MIMO Gaussian MAC," in The 5th International Symposium on Wireless Personal Multimedia Communications 2002, oct. 2002, pp. 130 - 134 vol.1.

[3] E. Jorswieck and H. Boche, "Transmission strategies for the MIMO MAC with MMSE receiver: average MSE optimization and achievable individual MSE region," IEEE Transactions on Signal Processing, vol. 51, no. 11 , pp. $2872-2881$, nov 2003.

[4] A. Del Coso and S. Simoens, "Distributed compression for MIMO coordinated networks with a backhaul constraint," IEEE Transactions on Wireless Communications, vol. 8, no. 9, pp. 4698-4709, 2009.

[5] A. Sanderovich, S. Shamai, and Y. Steinberg, "Distributed MIMO receiver - achievable rates and upper bounds," IEEE Transactions on Information Theory, vol. 55, no. 10, pp. 4419-4438, 2009.

[6] E. Candes and T. Tao, "Decoding by linear programming," IEEE Transactions on Information Theory, vol. 51, no. 12, pp. 4203-4215, Dec. 2005.

[7] J. Haupt and R. Nowak, "Signal reconstruction from noisy random projections," IEEE Transactions on Information Theory, vol. 52, no. 9, pp. 4036 - 4048, Sep. 2006.

[8] D. Baron, M. F. Duarte, M. B. Wakin, S. Sarvotham, and R. G. Baraniuk, "Distributed compressive sensing," CoRR, 2009, http://arxiv.org/abs/0901.3403.

[9] S. Corroy and R. Mathar, "Compressive forwarding for jointly sparse signals in amplify-and-forward gaussian relay networks," in IEEE International Conference on Communications (ICC 2011), june 2011. 